\title{
Milchprodukte aus biologisch-dynamischer Herkunft führen zu einer vorteilhafteren Fettzusammensetzung der menschlichen Muttermilch
}

\author{
Ana Paula Simões-Wüsta, lukas Rista, André Muellerb, Hans Steinhartb, \\ MACHTELD HUBERc, CAREL THIJS ${ }^{d}$ \\ a) Forschungsabteilung des Paracelsus-Spitals Richterswil, Bergstraße $16, \mathrm{CH}-8805$ Richterswil, Schweiz \\ b) Abteilung für Lebensmittelchemie, Universität Hamburg, Grindelallee 117, D-20146 Hamburg, Deutschland \\ c) Louis Bolk Institut, Hoofdstraat 24, NL-3972 LA Driebergen, Niederlande \\ d) Abteilung Epidemiologie, Forschungsinstitut Maastricht für Ernährung und Toxikologie, (Nutrim), \\ und Pflege und Gesundheitswesen Forschungsinstitut (Caphri), Universität Maastricht, PO Box 616, NL-6200 MD Maastricht, Niederlande
}

Milchprodukte aus biologisch-dynamischer Herkunft führen zu einer vorteilhafteren Fettzusammensetzung der menschlichen Muttermilch - Zusammenfassung

In unserer früheren Studie konnten wir zeigen, dass der Verzehr biologischer Milchprodukte durch stillende Mütter zu einem erhöhten Gehalt an konjugierten Linolsäure-Isomeren in der Muttermilch führt, was sich positiv auf die Gesundheit des Säuglings auswirken kann. Nun wurde der Einfluss biologischdynamischer Milchprodukte in der Diät auf den konjugierten Linolsäure-Isomerengehalt in der menschlichen Muttermilch untersucht. Der Anteil an Rumensäure (das am meisten vorhandene Isomere) in der Muttermilch der Frauen, die biologisch-dynamische Milchprodukte zu sich nahmen, war statistisch signifikant höher ( $n=64,0,323 \%$ vom gesamten Fett) als derjenige der sich konventionell ernährenden Mütter ( $n=175,0,254 \%$ ). Dabei zeigte die Muttermilch der Frauen, die biologische (aber nicht biologisch-dynamische) Milchprodukte zu sich nahmen, einen dazwischenliegenden Wert ( $n=44,0,279 \%)$. Die Variationen in dem Rumensäuregehalten wurden von ähnlichen Schwankungen in den Gehalten der entsprechenden Vorstufe, der trans-Vaccensäure, begleitet. Der Gehalt an Elaidinsäure, die in teilgehärteten pflanzlichen Ölen in hohen Mengen vorkommt, verhielt sich umgekehrt.

Ein möglicher Beitrag biologisch-dynamischer Milchprodukte zur Verbesserung der Gesundheit wird kurz besprochen.

\section{- Schlüsselwörter}

konjugierte Linolsäure

Rumensäure

trans-Vaccensäure

menschliche Muttermilch

biologische Ernährung

biologisch-dynamische Ernährung
Dairy products of biodynamic origin lead to a more favourable fat composition of human milk

\section{- Abstract}

Our previous work showed that the incorporation of organic dairy products in the maternal diet leads to increased contents of conjugated linoleic acid isomers in human breast milk, which might positively affect infant's health. Now, the effect of biodynamic - a special form of organic-dairy products in diet on the level of conjugated linoleic acid isomers in human breast milk has been analysed. The content of rumenic acid (the most common isomer of conjugated linoleic acids) in breast milk was higher in the women consuming biodynamic products ( $n=64$, $0.323 \%$ of total fat) than in the women consuming a conventional diet ( $n=175,0.254 \%$ ). The group of women consuming other dairy products including organic (but not biodynamic) showed an intermediate value $(n=44,0.279 \%)$. The levels of transvaccenic acid, a rumenic acid-precursor, paralleled those of rumenic acid, whereas the opposite happened with those of elaidic acid, whose levels are often high in partially hydrogenated fats. A likely contribution of biodynamic milk products to improve human health is briefly discussed.

\section{- Keywords}

Conjugated linoleic acid

Rumenic acid

Trans-vaccenic acid

Human milk

Organic nutrition

Biodynamic nutrition 


\section{Hintergrund}

$\mathrm{D}$ ie biologische Produktion von Nahrungsmitteln gemäßVerordnung EWG 834/2007 (EU-Öko-Verordnung) untersagt die Verwendung der Konservierung mittels ionisierender Strahlung sowie von chemisch-synthetischen Dünger- und Pflanzenschutzmitteln, vorbeugend applizierten Tierantibiotika, genetisch verändertem Saat- und Pflanzgut sowie den Einsatz anderer als den für Bioprodukte zugelassenen Zusatzstoffen'. Umfassende Studien der letzten Jahre lassen vermuten, dass sich die biologische Produktion von Lebensmitteln positiv auf deren ernährungsphysiologische Zusammensetzung auswirkt. Ergebnisse der niederländischern Geburt-Kohortenstudie KOALA² deuten darauf hin, dass zweijährige Kinder, die sich in Bezug auf Milchprodukte konsequent biologisch ernährten, ein (statistisch) bedeutend tieferes Risiko verzeichneten, Ekzeme zu entwickeln (1). Nach unserer Kenntnis handelt es sich hierbei um das erste Beispiel eines gesundheitsfördernden Effekts von einem biologischen Nahrungsmittel.

Dass die Produktionsart die Lebensmittelqualität beeinflussen kann, wurde besonders gut im Fall der Fettzusammensetzung von Kuhmilch untersucht. Mehrere Studien zeigten, dass Kuhmilch von Biobauernhöfen höhere Gehalte an konjugierter Linolsäure als konventionell produzierte Milch $(2,3)$ besitzt. Gleichermaßen weist das Fleisch von Wiederkäuern einen höheren Gehalt an konjugierter Linolsäure auf, wenn die Tiere mit Gras gefüttert wurden, wie in der biologischen Viehhaltung üblich (4). Konjugierte Linolsäure-Isomere bilden eine besondere Unterklasse der Fettsäuren, welche gemäß Daten aus Invitro-Experimenten und Tierversuchen die menschliche Gesundheit positiv beeinflussen können (5).

Da Wiederkäuerprodukte - Milch und Fleisch - die Hauptquelle konjugierter Linolsäure für Menschen darstellen, stellten wir die Hypothese auf, dass der Gehalt an konjugierter Linolsäure in der Milch stillender Frauen durch eine Diät mit erhöhtem Anteil biologischer Wiederkäuerprodukte gesteigert werden könnte. In unserer früheren Arbeit (6) wurde der Gehalt an konjugierter Linolsäure und trans-Vaccensäure in Milchproben von 310 stillenden Müttern analysiert, welche an der GeburtKohortenstudie KOALA teilnahmen. Die Studienteilnehmerinnen wurden nach dem Konsum konventioneller oder biologischer Produkte (Milch und Wiederkäuerfleisch) klassifiziert. Der Gehalt an Rumensäure und an trans-Vaccensäure in der Muttermilch nahm beim Übergang von einer konventionellen zu einer teilbiologischen zu einer strikt biologischen Diät zu. Umgekehrt war der Elaidinsäuregehalt, der häufig in teilgehärteten Ölen erhöht ist, tiefer in der biologischen Gruppe. Dies könnte gesundheitliche Auswirkungen auf die Neugeborenen zur Folge haben, da teilgehärtete Öle bekanntlich das Risiko von Herzkreislauferkrankungen vergrößern $(7,8)$.

Unter den Biobauernhöfen sind die biologisch-dynamischen Höfe als integrierte Einheiten strukturiert. Die durch einen Impuls von Rudolf Steiner 1924 initiierte biologisch-dynamische Landwirtschaft (9) sieht den Ge- brauch tierischer und mineralischer Stoffe und die Berücksichtigung irdisch-kosmischer Kräftezusammenhänge vor, um den Pflanzenbau und die Tierhaltung zu verbessern ${ }^{3}$. Ein Großteil der Frauen, die für die KOALAStudie Milchproben spendeten, nahmen Milchprodukte biologisch-dynamischer Herkunft zu sich. In jetziger Auswertung wurden die Rumensäure-, die trans-Vaccensäure- und die Elaidinsäuredaten aus der Milch dieser Frauen - die eine biologisch-dynamische Ernährungsweise hatten - im Vergleich zu den entsprechenden Daten der Frauen mit konventioneller und biologischer (aber nicht biologisch-dynamischer) Ernährungsweise bezüglich Milchprodukten analysiert.

\section{Methoden}

Muttermilchproben wurden durch stillende Teilnehmerinnen der KOALA-Studie, einer prospektiven GeburtKohortenstudie, gespendet (10). Zusammenfassend wurden 312 Frauen mit unterschiedlichen Ernährungsgewohnheiten (konventionell und alternativ) eingeschlossen. Jede Studienteilnehmerin gab eine Muttermilch probe einen Monat postpartum ab. Die Studie wurde von der Medizinischen Ethikkommission der Universität und des Universitätsspitals Maastricht, Niederlande, genehmigt. Sammeln, Lagerung und Verarbeitung der Milchproben wurden in einer anderen Veröffentlichung beschrieben (6). 0,2 ml Muttermilch wurde extrahiert und die enthaltenen Lipide nach Transmethylierung als Fettsäurenmethylester (Doppelbestimmung) mittels Gaschromatographie mit Flammenionisationsdetektor (GC-FID) und Silberionen-Hochleistungsflüssigkeitschromatographie (Ag+-HPLC), wie früher ausführlich beschrieben (6), analysiert. Für die GC-FIDAnalyse wurden ein Agilent 6890 Gaschromatograph (Agilent Technologies, Waldbronn, Deutschland) mit Split/Splitlos-Injektor bei $230{ }^{\circ} \mathrm{C}$, ein Flammenionisationsdetektor bei $260^{\circ} \mathrm{C}$, ein Autosampler und eine CP SIL 88 Säule (100 m, 0,25 mm, 0,2 $\mu \mathrm{m}$ Filmdicke; Varian, Darmstadt, Deutschland) verwendet. Konjugierte Linolsäureisomere wurden mittels Ag+-HPLC mit Diodenarraydetektion ( $\left.\mathrm{Ag}^{+}-\mathrm{HPLC}-\mathrm{DAD}\right)$ analysiert. Das System bestand aus einer isokratischen Merck-Hitachi L-60ooA-HPLC-Pumpe mit einem Waters 717 Autosampler (Waters, Eschborn, Deutschland) und einem bei einer Wellenlänge zwischen 210,4 und 395,4 nm betriebenen Waters 996 Diodenarray-Detektor. Drei Chromspher5-Lipid-Säulen ( $250 \mathrm{~mm} \times 4,6 \mathrm{~mm}, 5 \mu \mathrm{m}$, Varian) wurden in Serie geschaltet und mit einer $50 \mathrm{~mm} \times$ 4,6 $\mathrm{mm}$ Vorsäule aus demselben Säulenmaterial verwendet. Die Datenauswertung erfolgte mittels Agilent ChemStation (GC-FID) bzw. Waters Millenium. Der mittlere Gewichtsanteil der verschiedenen Fettsäuren in Prozent wurde für die nach Herkunft der Milchprodukte gruppierten Subjekte mit dem Student's T-Test ermittelt, um Unterschiede zwischen den Gruppen festzustellen (ohne Annahme von Streuungsgleichheit); ein Unterschied zwischen zwei Gruppen wurde als statistisch signifikant betrachtet, wenn $p \leq 0,05$ war. Alle statistischen Analysen
Anmerkungen ) http://ec.europa. eu/agriculture/organic/organic-farming_de 2) niederländisch für „Kinder, Eltern und Gesundheit: Lebensstile und genetische Konstitution GeburtKohortenstudie" 3) http://www. demeter.net/ 


\begin{tabular}{|c|c|c|c|c|}
\hline Gruppe & $\mathbf{n}$ & Rumensäure & trans-Vaccensäure & Elaidinsäure \\
\hline $\begin{array}{l}\text { Biologisch- } \\
\text { dynamische } \\
\text { Milchprodukte }\end{array}$ & 64 & $0,323 \pm 0,0128^{*}$ & $0,584 \pm 0,0257^{*}$ & $0,487 \pm 0,0232^{*}$ \\
\hline $\begin{array}{l}\text { Andere } \\
\text { biologische } \\
\text { Milchprodukte }\end{array}$ & 44 & $0,279 \pm 0,0160$ & $0,545 \pm 0,0307$ & $0,526 \pm 0,0375$ \\
\hline $\begin{array}{l}\text { Konventionell } \\
\text { hergestellte } \\
\text { Milchprodukte }\end{array}$ & 175 & $0,254 \pm 0,0052$ & $0,479 \pm 0,0150$ & $0,606 \pm 0,0188$ \\
\hline $\begin{array}{l}\text { Keine } \\
\text { Milchprodukteb }\end{array}$ & 27 & $0,230 \pm 0,0151$ & $0,464 \pm 0,0355$ & $0,596=$ \\
\hline
\end{tabular}

Die Daten werden als Durchschnitt \pm SEM gezeigt.

a inklusive biologische, aber exklusive biologisch-dynamische Milchprodukte

$p<0,001$, im Vergleich mit Studienteilnehmerinnen, die konventionell hergestellte Milchprodukte konsumiert haben

b haben Milchprodukte vermieden oder nicht konsumiert

wurden in SPSS 12.0 für Windows (www.spss.com) durchgeführt.

Der Ernährungsfragebogen war Teil eines Studienfragebogens, der in der 34 . Schwangerschaftswoche ausgefüllt wurde (10). Der Ernährungsfragebogen umfasste ca. 160 Lebensmittel, für welche die Aufnahmefrequenz und Portionsgröße geschätzt werden mussten. Außerdem wurde Information bezüglich Herkunft (konventionell, biologisch, biologisch-dynamisch) der verschiedenen Lebensmittelgruppen gesammelt.

\section{Resultate}

Insgesamt konnten 310 Studienteilnehmerinnen (Fettsäurebestimmung war nur für 310 der 312 Frauen vollständig) in die Analyse eingeschlossen werden, die meisten davon ( $n=175$ ) mit einer konventionellen Diät. Demgegenüber gaben 64 Frauen an, mindestens einige biologisch-dynamische und 44 andere biologische (exklusiv biologisch-dynamische) Milchprodukte zu konsumieren. Siebenundzwanzig Frauen aßen keine Milchprodukte. Der Fragebogen fragte gezielt nach Milch- und Wiederkäuerprodukten, da diese die Hauptquelle von konjugierter Linolsäure für Menschen darstellen. Obwohl eine große Anzahl Frauen biologisch-dynamische Milchprodukte zu sich nahm $(n=64)$, aß nur die Hälfte davon auch biologisch-dynamisches Fleisch $(n=32)$ und weniger noch nur biologisch-dynamisches Fleisch, aber keine Milchprodukte $(n=12)$. Deshalb werden nur die Daten über die biologisch-dynamischen Milchprodukte gezeigt.

Tab. 1 zeigt, dass der Rumensäuregehalt in der Muttermilch der Frauen, die biologisch-dynamische Milchprodukte zu sich nahmen, statistisch signifikant höher war (0,323\% des Gesamtfetts, $p<0,001$ ) als derjenige der Mütter mit konventioneller Ernährungsweise (0,254\%). Obwohl statistisch nicht signifikant, konnte ein Unterschied zwischen dem Rumensäuregehalt in der Milch der Mütter mit einer biologisch-dynamischen Diät $(0,323 \%)$ und demjenigen der Frauen festgestellt werden $(0,279 \%)$, die angaben, andere biologische (aus- schließlich biologisch-dynamische) Milchprodukte zu sich zu nehmen. Der Rumensäuregehalt war am tiefsten (0,230\%) in der Gruppe von Frauen, die Milchprodukte ganz vermieden, was die Bedeutung der Milchprodukte als Rumensäure-Lieferanten bestätigt.

Erwartungsgemäß entsprachen die Resultate für die trans-Vaccensäure denjenigen für die Rumensäure. Wiederum wurde die höchste trans-Vaccensäure-Konzentration in der Muttermilch von Frauen festgestellt, die biologisch-dynamische Milchprodukte zu sich nahmen (0,584\%, p<0,001 im Vergleich zu konventionellen Milchprodukten), gefolgt von den Müttern, die andere biologische Milchprodukte verwendeten (0,545\%). Die trans-Vaccensäuregehalte in der Gruppe mit konventioneller Ernährungsweise (0,479\%) und in der Gruppe ohne Milchprodukte $(0,464 \%)$ waren die niedrigsten und miteinander vergleichbar.

Ebenfalls Tab. 1 zu entnehmen ist, wie sich der Elaidinsäuregehalt zwischen den verschiedenen Gruppen unterscheidet. Der Anteil von dieser in teilgehärteten Ölen reichlich vorhandenen Fettsäure war statistisch signifikant tiefer in der Gruppe mit biologisch-dynamischen Essgewohnheiten als in der Gruppe, die sich konventionell ernährte $(0,487 \%$ versus $0,606 \%, p<0,001)$. In der Gruppe, die andere biologische Milchprodukte verwendete, zeigte der Elaidinsäuregehalt keinen signifikanten Unterschied zu der konventionellen Gruppe.

\section{Diskussion}

Durch die hier gezeigten Resultate wird ersichtlich, dass die Aufnahme biologisch-dynamischer Milch und Milchprodukte durch stillende Mütter zu erhöhten Anteilen von Rumensäure und trans-Vaccensäure sowie zu einem tieferen Elaidinsäuregehalt in der Muttermilch relativ zur Aufnahme konventionell hergestellter Milch führt. Die festgestellten Unterschiede bestätigen unsere früheren Befunde über die Muttermilchzusammensetzung bei Frauen, die biologische bzw. konventionelle Milchprodukte verzehren.

Während den konjugierten Linolsäuren und deren Vorstufe trans-Vaccensäure eine Vielzahl positiver Wirkungen nachgesagt wird (günstiges Verhältnis von Fettgewebe zur Muskelmasse sowie anticarcinogene, antiatherosklerotische, antidiabetische und immunmodulierende Effekte (11)), werden die durch einen hohen Gehalt an Elaidinsäuregehalt gekennzeichneten, teilgehärtete Ölen verdächtigt, zu pathologischen Zuständen wie ischämischer Herzerkrankung (8) und Typ-2-Diabetes (7) beizutragen. Dieser Verdacht veranlasste einige Regierungen, wie z. B. die dänische (8) und die schweizerische (12), die Gesamtmenge der entsprechenden Fettsäuren in den Lebensmitteln gesetzlich zu begrenzen.

\section{Schlussfolgerung}

Sowohl die in der Muttermilch feststellbaren höheren Konzentrationen an Rumensäure und trans-Vaccensäure wie auch der tiefere Gehalt an Elaidinsäure, welche durch den Verzehr biologisch-dynamischer Milchprodukte erreicht werden, können einen positiven Beitrag zur Gesundheit der Säuglinge liefern. Im Vergleich 
zu den herkömmlichen Bioprodukten scheinen Milchprodukte aus biologisch-dynamischer Produktion zu den höchsten Gehalten an konjugierter Linolsäure und dem niedrigsten Gehalt an Elaidinsäure in der Muttermilch zu führen.

\section{Danksagung}

Wir sind Chantal Wälchli für die Übersetzung des originalen Texts ins Deutsche sehr dankbar. Diese Arbeit wurde durch die niederländische Organisation für Gesundheit, Forschung und Entwicklung (ZonMw, Niederlande), die Royal Friesland Foods (Niederlande), die Triodos Stiftung (Niederlande), den UDEA organic retail (Niederlande), die Biologica organisation for organic farming and food (Niederlande) und den Konsumentenverein zur Förderung der biologisch-dynamischen Landwirtschaft Zürich (Schweiz) finanziell unterstützt.

\section{Korrespondenzadresse:}

Dr. A. Paula Simões-Wüst

Forschungsabteilung des Paracelsus-Spitals Richterswil

Bergstraße 16

$\mathrm{CH}-8805$ Richterswil, Schweiz

Telefon: + 41 (o)44/787 2493

Fax: +41 (o) 44/7872351

simoes@paracelsus-spital.ch

\section{Literatur}

1 Kummeling I, Thijs C, Huber $M$, et al. Consumption of organic foods and risk of atopic disease during the first 2 years of life in the Netherlands. Br J Nutr 2008; 99: 598-605

2 Bergamo P, Fedele E, lannibelli L, et al. Fat-soluble vitamin contents and fatty acid composition in organic and conventional Italian dairy products. Food Chemistry 2003; 82: 625-631 3 Jahreis G, Fritsche J, Steinhart $\mathrm{H}$. Conjugated linoleic acid in milk fat: high variation depending on production system Nutr Res 1997; 17: 1479-1484 4 Dannenberger D, Nuernberg $\mathrm{G}$, Scollan N, et al. Effect of diet on the deposition of n-3 fatty acids, conjugated linoleic and C18:1trans fatty acid isomers in muscle lipids of German Holstein bulls.J Agric Food Chem 2004; 52: 6607-15

5 Bhattacharya A, Banu J, Rahman M, et al. Biological effects of conjugated linoleic acids in health and disease. J Nutr Biochem 2006; 17: 789-810 6 Rist L, Mueller A, Barthel C, et al. Influence of organic diet on the amount of conjugated linoleic acids in breast milk of lacta ting women in the Netherlands. Br J Nutr 2007; 97: 735-43
7 Odegaard A, Pereira M. Trans fatty acids, insulin resistance, and type 2 diabetes. Nutr Rev 2006; 64: 364-372

8 Stender S, Dyerberg J. Influence of trans fatty acids on health Ann Nutr Metab 2004; 48: 61-6 9 Koepf H, Plato B v. Die biologisch-dynamische Wirtschaftsweise im 20. Jahrhundert.

Dornach: Verlag am Goetheanum, 2001

10 Kummeling I, Thijs C, Penders $J$, et al. Etiology of atopy in infancy: the KOALA Birth Cohort Study. Pediatr Allergy Immunol 2005; 16: 679-84

11 Belury M. Dietary conjugated linoleic acid in health: physiological effects and mechanisms of action. Annu Rev Nutr 2002; 22: 505-531

12 Richter EK, Albash Shawish K, Scheeder MRL, et al. Trans fatty acid content of selected Swiss foods: The TransSwissPilot study. J Food Comp Anal 2009; http://dx.doi.org/10.1016/j.jfca. 2009.01.007 